Nonlinear Phenomena in Complex Systems, vol. 23, no. 4 (2020), pp. 405 - 413

\title{
Post-Quantum Group-Oriented Authentication in IoT
}

\author{
E. B. Aleksandrova, ${ }^{*}$ A. A. Shtyrkina, ${ }^{\dagger}$ and A. V. Yarmak ${ }^{\ddagger}$ \\ Institute of Applied Mathematics and Mechanics, Cybersecurity Department, \\ Peter the Great St. Petersburg Polytechnic University (SPbPU), \\ 29 Polytechnicheskaya Str, 195251 St.Petersburg, Russian Federation
}

(Received 19 August, 2020)

The Internet of Things may include sensors, actuators, analyzers, logical controllers, which together form distributed network of heterogeneous devices. Group-based approach to authentication may be relevant when the number of nodes is very large and there are constrained devices among them. Post-quantum schemes as candidates in NIST competition are considered. Lattices are chosen as best candidates for building group-oriented schemes for IoT due to high performance, relatively small key sizes, well researched mathematical problem. To give flexibility to the lattice-based group-oriented authentication protocols, the basis delegation mechanism was considered as an approach that takes into account the hierarchy in the Internet of Things systems.

AMS Subject Classification: 81P94, 94A62

Keywords: post-quantum cryptography, group-oriented authentication, digital signature, latticebased cryptography, information security

DOI: https://doi.org/10.33581/1561-4085-2020-23-4-405-413

\section{Introduction}

The concept of the Internet of Things (IoT) involves the union of physical elements into a network to interact with each other or with the external environment. This paradigm opens up opportunities for construction essentially new systems, where physical and information processes are integrated closely.

In general, an IoT system includes devices that differ in computational power, functionality and roles in a common network. The following types of devices are distinguished:

- sensors, with the help of which information is collected in real time for further processing and storage;

- actuators, by means of which the IoT system affects the environment;

- gateways, which are designed for primary

\footnotetext{
${ }^{*}$ E-mail:helen@ibks.spbstu.ru

${ }^{\dagger}$ E-mail:anna \_sh@ibks.spbstu.ru

${ }^{\ddagger}$ E-mail:yarmak. av@ibks. spbstu.ru
}

processing of information coming from sensors;

- controllers, which perform complex processing of data coming from devices below the hierarchy and make decisions on further organization of processes, including transfer of commands to actuators.

The IoT concept is actively implemented in various spheres of human activity. Bright examples of IoT applications are: transport infrastructure, in which vehicle sensors (MANET, VANET, FANET) act as devices; medical care, where devices collect and aggregate information about human health; smart manufacturing, where devices exchange information to automate industrial processes; smart home, where the devices control the processes inside a residential area.

Whatever area the IoT concept is implemented in, data security is an important issue. Confidentiality, integrity and accessibility are important both in the health care sector, where sensitive information circulates, and in transport and industrial infrastructures. For 
example, disruptions to process security can have catastrophic consequences for the government, the environment, and human health.

The problem of ensuring security in IoT systems is complicated by a number of features:

- the rapid growth in the number of network devices;

- distribution of nodes;

- heterogeneity of device characteristics (computing power, functionality, roles, supported protocols);

- dynamic topology (MANET, VANET, FANET).

One of the most important problems in ensuring IoT systems security is to maintain secure communication between nodes by authenticating information circulating within the system. A specific of IoT systems is that a set of devices is combined into a hierarchical structure, in which low-power devices (sensors, actuators, etc.) are located at the lower level of the hierarchy, and the higher levels contain devices with greater power and the ability to perform complex operations, including monitoring of devices below the hierarchy. Since the number of devices in modern IoT systems is large, it is proposed to consider the problem of providing authentication, considering not specific devices, but entire groups of devices. In this regard, a suitable cryptographic protocol is group-oriented authentication schemes. As it shown in Chapter 2 , one of the most promising mathematical problems for constructing cryptographic protocols, including devices with low power, is the lattice theory problems. For latticebased cryptosystems, there is a basis delegation mechanism that allows one to take into account both the dynamically changing distribution of elements in IoT systems and the hierarchical organization of devices in these systems.

\section{Group-oriented authentication approaches}

Digital signature is one of the most common cryptographic mechanisms of message authentication. Existing standards in the field of digital signatures, and also modern researches, including postquantum mechanisms of authentication, are concentrated on signature schemes, where a single user acts as the signing party. In case when the message should be signed by a group of users the simplest decision consists in association of individual signatures of group members. As the group size grows, both the time of signature generation and its verification will increase, which is unacceptable in distributed systems like IoT, with many devices and a variable group composition.

For the first time, the need for grouporiented cryptography has been highlighted in Desmedt [1], which includes such aspects of group-oriented cryptography as user anonymity, presence of a trusted party, exclusion a group member, and others. Later, this concept was developed in the threshold digital signature scheme [2], which uses the scheme of secret sharing for distribution of signature key between all group members so that a certain threshold value of system members is required for authentication. As with other cryptographic algorithms based on secret sharing schemes, a threshold signature scheme requires a trusted party, the dealer responsible for generating partial secrets. At the same time, Chaum and van Heyst proposed the concept of a group signature scheme [3], which is a particular case of a threshold signature for a threshold value of 1 . As part of a group signature, a group member can sign messages on behalf of the group, while maintaining anonymity. To manage the group, the issuing manager and opening manager are introduced, which are responsible for including members in the group and open the identity of the signer. Thus, the anonymity of the user can be revoked in case of conflict.

Ring signature, first proposed in [4], allows 
to sign a message from a group of users, ring members, observing the property of complete anonymity. A pair is associated with each ring member: a signature key and verification key. The simplest ring signature scheme consists of generating signature for a message using the given public keys of the ring members and the private key of the signature author, as well as verifying the message signature.

The use of collective signature is justified if it is necessary to form a signature by all members of a group or some its subset. The resulting multisignature is equivalent to combining the individual signatures of the group members, however, as a rule, it has a fixed length less than the total size of the individual signatures, and can also be verified in constant time. A special case of a multisignature is a structural, or hierarchical, signature that takes into account the order in which the signature is formed. If, in the case of multisignature, all group members sign one message, then the aggregated signature is a single short signature, which is equivalent to combining the signatures of the group members for different messages.

The main methods of group authentication are presented in Table I. It should be noted that the considered concepts can be added with additional properties, for example, blindness (blinded), undeniability, etc., as well as implement a hybrid approach, for example, a threshold ring signature.

Thus, the aim of group authentication is to optimize the process of organizing secure interaction, taking into account the specifics of the IoT by processing authentication data at the level not of specific nodes, but of subsystems and groups of devices aggregated according to some criteria (technological subprocesses in case of industrial IoT, location, computational characteristics, etc.). The application of this approach is associated with the need to solve a number of additional tasks, such as ensuring the anonymity of nodes in order to protect them from targeted exposure, revoking the right to sign on behalf of the group if a node is failed or compromised, updating data for group members and devices, etc.

In particular, the use of ring signatures to ensure message authentication and access control in IoT is considered in [5-7]. For example, an authentication scheme based on an identity-based ring signature with unconditional privacy and enhanced privacy under full key exposure attack properties is proposed in [6]. A group of elliptic curve points is used as mathematical structure, and bilinear maps are used for the signature verifying. In [7], a scheme for access control in wireless sensor networks is proposed, based on modification of the signature protocol in a group of elliptic curve points.

Group signatures are also one of the promising areas for IoT security [8]. This approach allows the privacy and anonymity of the signer, which is relevant for IoT devices. For example, in [9] it is proposed to check the validity of messages sent on the VANET using a group signature and a pseudonym system. The lightweight pseudonym with trapdoor mechanism eliminates the need of Certificate Revocation List.

\section{Post-quantum cryptography}

It is known that development of quantum computer will break the modern asymmetric cryptosystem. Due to the fact that the largest corporations in the world are engaged in the development of the quantum computer prototype, the task of substitution of cryptographic protocols based on the problems of discrete logarithm and integer factorization is one of the most relevant in the modern scientific community.

In 2016, the NIST Institute announced a competition [10] aimed at developing effective cryptographic protection schemes based on the problems resistant to quantum computer attacks. In 2019, 26 algorithms, the finalists of the second round of the competition were announced. Among them there are:

- hash-based; 
Table 1: Group-oriented authentication approaches.

\begin{tabular}{|c|c|c|c|c|c|c|}
\hline Properties & \begin{tabular}{|l}
$\begin{array}{l}\text { Group } \\
\text { signature }\end{array}$ \\
\end{tabular} & \begin{tabular}{|l|}
$\begin{array}{l}\text { Ring } \\
\text { signature }\end{array}$ \\
\end{tabular} & $\begin{array}{l}\text { Threshold } \\
\text { signature }\end{array}$ & Multisignature & $\begin{array}{l}\text { Aggregate } \\
\text { signature }\end{array}$ & $\begin{array}{l}\text { Structured } \\
\text { signature }\end{array}$ \\
\hline Number of signers & One & One & $\begin{array}{l}t \text { signers, where } \\
t \text { is threshold } \\
\text { value }\end{array}$ & $\begin{array}{ll}\text { All group } & \text { or } \\
\text { subgroup } & \text { of } \\
\text { group members }\end{array}$ & $\begin{array}{l}\text { All group } \\
\text { members }\end{array}$ & $\begin{array}{l}\text { All group } \\
\text { members }\end{array}$ \\
\hline $\begin{array}{l}\text { Number } \\
\text { messages }\end{array}$ & One & One & One & One & Multiple & One \\
\hline Trusted party & \begin{tabular}{|l|} 
Yes (issuing \\
manager, \\
opening \\
manager
\end{tabular} & No & Yes & No & No & No \\
\hline Static/Dynamic & $\begin{array}{l}\text { Static, } \\
\text { dynamic }\end{array}$ & Dynamic & $\begin{array}{l}\text { Static, dynamic } \\
\text { with possibility } \\
\text { of change } \\
\text { threshold value }\end{array}$ & Static & Static & Static \\
\hline $\begin{array}{l}\text { Identification } \\
\text { signer }\end{array}$ & \begin{tabular}{|l|} 
Only with \\
opening \\
manager
\end{tabular} & No & No & Yes & $\begin{array}{l}\text { Yes } \\
\end{array}$ & Yes \\
\hline Hierarchy & No & No & No & Yes/No & Yes/No & Yes \\
\hline
\end{tabular}

- code-based;

- multivariate;

- lattice-based;

- isogeny-based.

Cryptosystems based on such problems have at least sub-exponential complexity for both quantum and classical computers.

The construction of hash-based cryptographic schemes is reduced to the assumption that the used hash function is resistant to finding collisions and/or preimages problems. This concept is well established and has the advantages of high performance and relatively short key lengths. Among the disadvantages of such schemes, one can single out the large size of the signature, as well as the lack of encryption protocols and establishing a common key. The use of hash-based cryptography to provide group authentication is limited to the work associated with one-time group signature [11] and sequential aggregate signature [12].

Some schemes are based on the complexity of complete linear codes decoding. This problem is well studied, and the NIST contesting schemes are among the most productive. However, there is no digital signature scheme among the NIST competition candidates based on this problem. Code-based cryptography is also used to build post-quantum group authentication schemes, including hybrid ones. For example, in [13], a scheme is presented that combines the properties of a threshold and a ring signature, where the signer is not a one user who forms a signature on behalf of a group, but a subset of t-out-of-N users. This approach also ensures the anonymity of the user group and does not allow the disclosure of information about which subset of the group generated the signature.

In multivariate cryptography, security is based on the fact that solving quadratic polynomials over a finite field, in the general case, is an NP-complete problem. This problem is well studied; its advantages include a relatively small signature size. Among the shortcomings, one can single out the large size of the keys, as well as the lack of encryption and common key generation algorithms among the candidates for the NIST competition. The multivariate-based 
cryptography problems are used to build schemes for group signatures [14], threshold signatures [15], ring signatures [16].

The security of lattice-based cryptosystems is based on a number of complex computational problems from lattice theory. Such problems include: Shortest Vector Problem, Shortest Linear Independent Vectors Problem, Approximate Shortest Vector Problem, Short Integer Solution, Learning with Errors. The problems of lattice theory are well studied. Cryptosystems based on lattice theory have a fairly high performance and are characterized by a small signature size. However, the selection of the parameters of this cryptosystems is a laborious process. Lattice problems are fairly well understood. Currently, there are many group-oriented authentication schemes based on these objectives. In [17], the authors propose a verifier local revocation group signature scheme based on the SIVP. [18] proposes a lattice-based ring signature whose security is based on the SIS problem. The paper [19] proposes a threshold ring signature based on the SIS problem.

The problem of isogeny computation is relatively young. It was first proposed to use isogeny to construct asymmetric cryptosystems in 2006 [20]. In 2010, for such a problem, a sub-exponential algorithm [21] was found for finding isogenies on quantum computer, therefore, cryptographic protocols are now being built on the problem of finding isogenies for supersingular elliptic curves. Such protocols are characterized by rather small key sizes and relatively good performance. Most of the work in the field of elliptic curve isogeny focuses on solving the key establishing methods, as well as message authentication schemes where a single user acts as a signer. Among the methods of grouporiented authentication, methods of threshold authentication [22], as well as hierarchical collective authentication [23], based on the problem of finding supersingular curves isogeny, have been proposed.

The following digital signature candidates entered the second round of the NIST competition: SHPHINCS + (hash-based), qTESLA (lattice-based), DILITHIUM (latticebased), LUOV (multivariate), Picnic-UR (hashbased), Picnic-FS (hash-based), Falcon (latticebased), MQDSS (multivariate).

Figure 1 shows the comparative characteristics of candidates in terms of the size of keys and signature (top) and operating time (low). DILITHIUM, FALCON, qTESLA showed some of the best results in almost all parameters. Their characteristics are shown in Figure 2. When comparing the algorithms, the results presented in Table II were obtained.

As can be seen from the comparison results, lattice-based schemes showed the best results for signature size and verification speed. These parameters are decisive when building cryptography schemes for IoT, since the amount of information sent in the network depends on the size of the signature, and the verification speed is critical for low-power devices. Moreover, the characteristics of lattice-based signatures (DILITHIUM, FALCON, qTESLA) are more balanced relative to others, where an improvement in one or two characteristics leads to a sharp increase in the third.

\section{Applying basis delegation for hierarchical multisignature}

The flexibility of protocols on elliptic curves, which allows constructing BLS and group signature, hierarchical identity-based encryption [24], is provided by bilinear map. A similar mechanism for lattices was proposed in 2009-2010 in $[25,26]$. The basis delegation mechanism allows using a short basis of a given lattice to generate a new short basis related to the lattice. In this case, the short basis is a cryptographic trapdoor, which allows consider the delegation of the basis as a convenient cryptographic primitive, for example, for building protocols for hierarchical systems.

Conventionally, such a mechanism can be visualized as a tree. Such a tree illustrates the hierarchy of entities in the system. In [26], a 


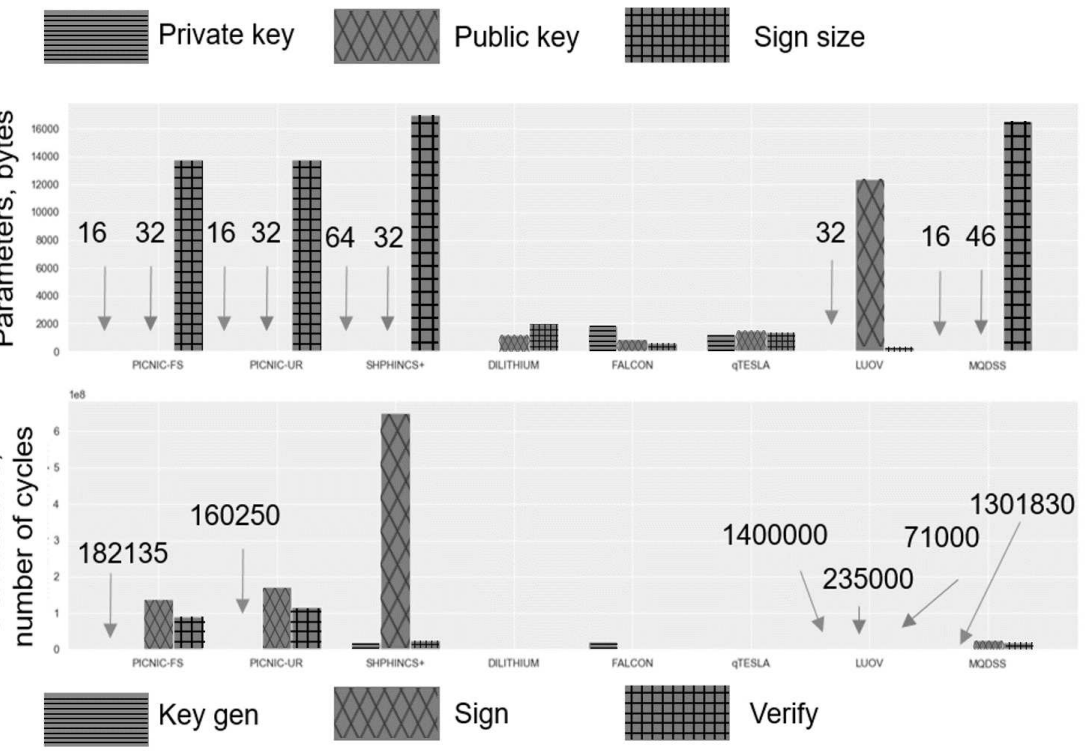

FIG. 1: Characteristics of NIST Round 2 candidates.

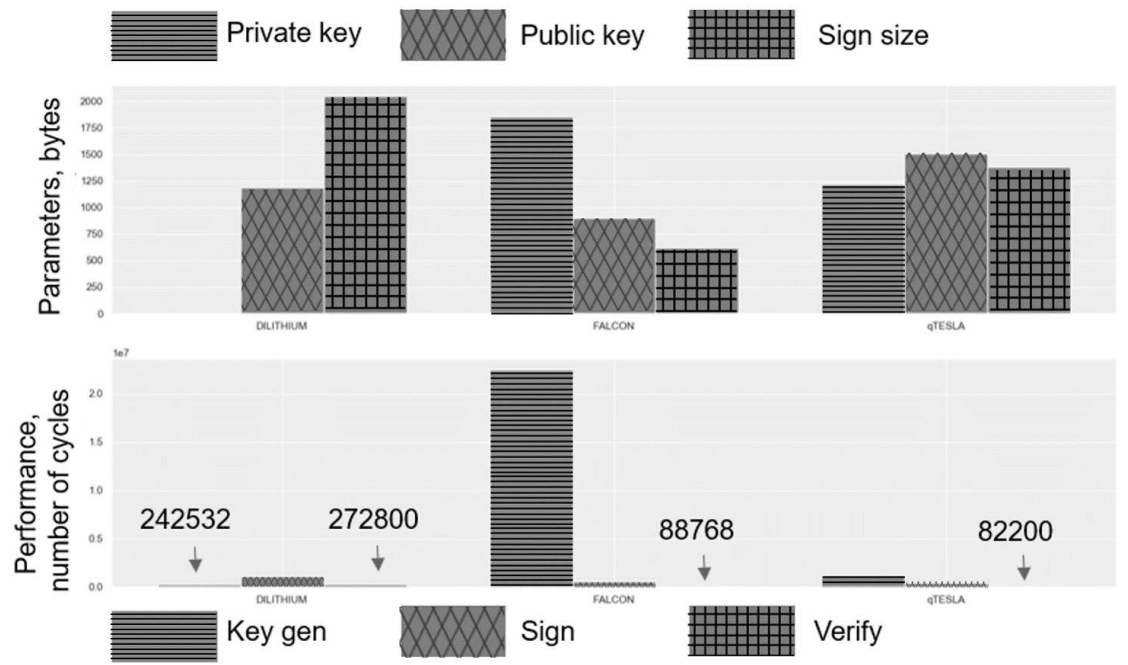

FIG. 2: Characteristics of DILITHIUM, FALCON, qTESLA.

tree is a set of hierarchically arranged lattices selected from a certain family, taking into account lattice-based assumptions. A characteristic of such structure is that the lattices located in the hierarchy below are higher-dimensional superlattice of its parent. In such a structure, the entity can generate the short basis of his children, but cannot restore the basis of his parents. Thus, the delegation process is one-way: a child node cannot use its short basis to restore the basis of its parent or its siblings.

For IoT such a hierarchical structure is preferred due to the network organization. In this case, gateways should be located at the root of the 
tree, controllers below in the hierarchy, sensors, actuators, etc. in the leaves. However, the increase in the size of private keys and authentication parameters as you descend from parent to child leads to the fact that low-power IoT devices, which are at the very bottom of the hierarchy, must have sufficient memory and performance. The solution to this problem is seen in basis delegation without dimension increase [27], when the dimensions of the parent and child lattices are the same.

Let us consider a basic matrix $A$ and a short basis $T_{A}$ of the lattice $\Lambda_{q}^{\perp}(A)$. A public matrix $R_{i}$ is associated with each node in the hierarchical structure. It allows based on the matrix $A$, matrix $B_{i}=A * R_{i}^{-1}$ using the BasisDel procedure described in detail in [27], to generate a short basis $T_{B_{i}}$ of the lattice $\Lambda_{q}^{\perp}\left(B_{i}\right)$ for a node that is a descendant of the root node. Thus, using known $A, B_{i}$, and $T_{B_{i}}$, it is difficult to reconstruct $T_{A}$, which is achieved due to the fact that the matrix $R_{i}$ has a "low norm"and SampleRwithBasis algorithm action from [27]. Generation of a short basis for the descendant located lower in the hierarchy, the matrix $B_{i j}$ is already determined by the relation $B_{i j}=B_{i} * R_{i j}^{-1}$ , and the corresponding short basis $T_{B_{i j}}$ can also be found using the same BasisDel polynomial algorithm, as shown in Figure 3.

Using the basis delegation mechanism, it is possible to organize the hierarchy of users in collective signature schemes. If tree structure is considered, the root of the tree corresponds to the main manager of the group, who has the most rights and controls and generates keys for the managers in the hierarchy below. Each local manager is responsible for generating keys for its subgroup (childs).

In terms of IoT, a Programmable Logic Controller (PLC) can act as a local manager, receiving data and transmitting a stream of commands to the underlying sensors and actuators. When a new sensor is connected to the group, the local manager performs the procedure for delegating the basis and generating keys for the new device.
In general, the public key of the general manager is matrix $A$, the public keys of other members of the group are matrices $B_{i}$, respectively. Matrix $R_{i}$ is associated with each group member and allows performing the basis delegation mechanism. The group master key is the short basis $T_{A}$ of the lattice $\Lambda_{q}^{\perp}(A)$, the key for other participants is the short basis $T_{B_{i}}$ of the lattice $\Lambda_{q}^{\perp}\left(B_{i}\right)$, respectively.

To generate an ordered signature, an individual lattice-based signature can be used, supplemented by a basis delegation mechanism. Each group member, in turn, generates a signature based on his private key, a short basis $T_{B_{i}}$, and then transfers the received partial signature to the next member. The next participant, using his private key and the received partial signature, generates a new signature, etc. Knowing the structure of the hierarchy and the public keys of the group members, the verifier checks the chain and the correctness of the signature.

The mechanism for binding partial messages depends on the underlying individual signature scheme. For example, for a hierarchy arranged as a one-way directional chain of nodes $R_{1}, R_{2}, \ldots, R_{n}$ ( $R_{1}$ is the bottom layer of the hierarchy, $R_{n}$ is the top layer of the hierarchy) the generation of an hierarchical multisignature begins with nodes at the bottom of the hierarchy. Each node computes the signature $s_{i}$ and passes it on to the higherlevel object.

Then for SIS-based scheme like GPV [28], signature for message $M$ generated by node $_{i-1}$, assotiated with matrix $R_{i-1}$, is a result of algorithm $s_{i-1}=$ SamplePre $\left(B_{i-1}, T_{B_{i-1}}, H\left(M\left\|R_{1}\right\| . .|| R_{i-1}\right), \sigma\right)$. The parent node verifies the received signature $s_{i-1}$ using the child's public key by verifying the correctness conditions for the GPV-signature: $B_{i-1} s_{i-1}=H\left(M\left\|R_{1}\right\| . . \| R_{i-1}\right)(\bmod q)$ and $\left\|s_{i-1}\right\|<6 n \log q$, where $n$ and $q$ - parameters of GPV-scheme. If the conditions are not met, the generation of the signature is terminated with informing of the overlying nodes. If the signature was formed correctly, then the parent 
Table 2: Best results among NIST Round 2 candidates.

\begin{tabular}{|l|l|l|l|}
\hline Shortest private key & $\begin{array}{l}\text { Picnic, } \\
\text { MQDSS }\end{array}$ & Fastest key generation & Picnic \\
\hline Shortest public key & $\begin{array}{l}\text { Picnic, } \\
\text { SPHINCS }+\end{array}$ & Fastest signature generation & LUOV \\
\hline Shortest signature size & Falcon & $\begin{array}{l}\text { Fastest } \\
\text { verification }\end{array}$ & qTesla \\
\hline
\end{tabular}

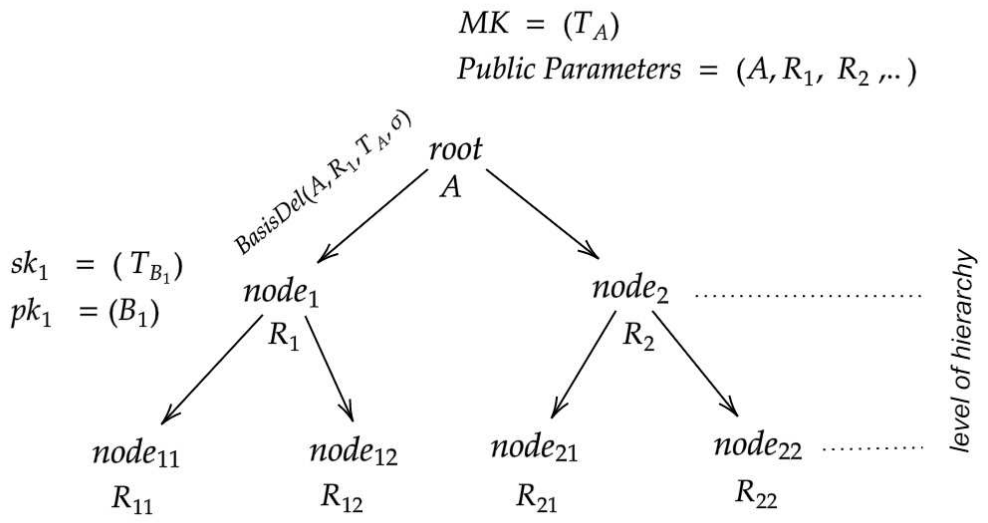

FIG. 3: Hierarchy with basis delegation.

node $R_{i}$ in a similar way, using its private key $T_{B_{i}}$, compute the signature, using the concatenation of the message with the chain of $R_{1}, R_{2}, \ldots, R_{i-1}, R_{i}$-matrices as an argument of hash function $H$.

This scheme can be extended to a hierarchy in the form of a tree or other structure, however, it requires specifying the ordering for nodes located at the same level.

\section{Conclusion}

Analyzing the typical architecture of the IoT, one can conclude that group authentication is one of the promising approaches to organizing secure interaction between IoT nodes by taking into account the network topology of IoT systems, including dynamic topology and resource distribution.

Among the candidates' signatures, the 2nd round of the NIST competition for the standard of post-quantum cryptography, lattice schemes demonstrate the smallest value of the parameter and signature lengths.

In cryptosystems based on discrete logarithm problem, the flexibility of group authentication protocols is ensured by the use of bilinear map, which simplifies the signature verification. In lattice cryptosystems, the basis delegation can also be used to take into account the hierarchy of group members. A further direction of research is related to the detalization of the mechanism of hierarchical authentication on lattices based on delegation of the basis in the IoT.

\section{Acknowledgment}

The reported study was funded as the part of the State Task for Basic Research (code 
of theme: 0784-2020-0026); suppl. agreement to the Agreement for the financial support
No.075-03-2020-158/2, 17.03.2020 (internal No.075-GZ/SCH4575/784/2).

\section{References}

[1] Y. Desmedt, in Conference on the Theory and Application of Cryptographic Techniques (Springer, 1987), pp. 120-127.

[2] Y. Desmedt and Y. Frankel, in Annual International Cryptology Conference (Springer, 1991), pp. 457-469.

[3] D. Chaum and E. Van Heyst, in Workshop on the Theory and Application of of Cryptographic Techniques (Springer, 1991), pp. 257-265.

[4] R. L. Rivest, A. Shamir, and Y. Tauman, in International Conference on the Theory and Application of Cryptology and Information Security (Springer, 2001), pp. 552-565.

[5] L. Malina, J. Hajny, P. Dzurenda, and S. Ricci, in ICETE (2) (2018), pp. 692-697.

[6] J. Li, Z. Zhang, L. Hui, and Z. Zhou, IEEE Access 8, 39689 (2020).

[7] D. He, J. Bu, S. Zhu, S. Chan, and C. Chen, IEEE Transactions on wireless communications 10, 3472 (2011).

[8] A. Wasef and X. Shen, in 2010 IEEE International Conference on Communications (IEEE, 2010), pp. 1-5.

[9] U. Rajput, F. Abbas, H. Eun, and H. Oh, IEEE Access 5, 12014 (2017).

[10] N. P.-Q. Cryptography-Workshops (Technical report, NIST, 2017. https://csrc.nist.gov/Projects/post-quantumcryptography/workshops-and-timeline, 2017).

[11] R. El Bansarkhani and R. Misoczki, in International Conference on Post-Quantum Cryptography (Springer, 2018), pp. 441-463.

[12] A. A. Yavuz and P. Ning, in 2009 6th Annual International Mobile and Ubiquitous Systems: Networking \&s Services, MobiQuitous (IEEE, 2009), pp. 1-10.

[13] H. Assidi, E. B. Ayebie, and E. M. Souidi, Journal of information security and applications 45, 52 (2019).

[14] G. Yang, S. Tang, and L. Yang, in International Conference on Information Security Practice and Experience (Springer, 2011), pp. 181-195.
[15] Y. Tao, Y. Yang, Z. Li, and X. Zheng, in 2012 IEEE International Conference on Intelligent Control, Automatic Detection and High-End Equipment (IEEE, 2012), pp. 114-118.

[16] S. Wang, R. Ma, Y. Zhang, and X. Wang, Computers \& Mathematics with Applications 62, 3973 (2011).

[17] A. Langlois, S. Ling, K. Nguyen, and H. Wang, in International Workshop on Public Key Cryptography (Springer, 2014), pp. 345-361.

[18] S. Wang et al., arXiv preprint arXiv:1405.3177 (2014).

[19] P.-L. Cayrel, R. Lindner, M. Rückert, and R. Silva, in International Conference on Cryptology and Information Security in Latin America (Springer, 2010), pp. 255-272.

[20] A. Rostovtsev and A. Stolbunov, IACR Cryptol. ePrint Arch. 2006, 145 (2006).

[21] A. Childs, D. Jao, and V. Soukharev, Journal of Mathematical Cryptology 8, 1 (2014).

[22] O. Pendrikova and E. Aleksandrova, Nedelya nauki SPbPU)(Saint-Petersburg: 2019) (2019).

[23] E. Alexandrova, M. Poltavtseva, and A. Yarmak, in SHS Web of Conferences (EDP Sciences, 2018), vol. 44, p. 00007.

[24] C. Gentry and A. Silverberg, in International conference on the theory and application of cryptology and information security (Springer, 2002), pp. 548-566.

[25] D. Cash, D. Hofheinz, and E. Kiltz, IACR Cryptol. ePrint Arch. 2009, 351 (2009).

[26] D. Cash, D. Hofheinz, E. Kiltz, and C. Peikert, in Annual International Conference on the Theory and Applications of Cryptographic Techniques (Springer, 2010), pp. 523-552.

[27] S. Agrawal, D. Boneh, and X. Boyen, in Annual Cryptology Conference (Springer, 2010), pp. 98115.

[28] C. Gentry, C. Peikert, and V. Vaikuntanathan, in Proceedings of the fortieth annual ACM symposium on Theory of computing (2008), pp. 197-206. 